\title{
CARACTERIZAÇÃO HIDRÁULICA E TÉCNICA DE TUBOS EMISSORES NÃO REGULADOS $^{1}$
}

\author{
ALEXANDRE BARCELLOS DALRI ${ }^{2 *}$, CARLOS JESUS BACA GARCIA ${ }^{3}$, LUIZ FABIANO PALARETTI $^{4}$, \\ JOSÉ RENATO ZANINI ${ }^{4}$, ROGÉRIO TEIXEIRA DE FARIA ${ }^{4}$
}

\begin{abstract}
RESUMO - Este trabalho teve como objetivo caracterizar e avaliar sete modelos de tubos emissores não regulados e de fluxo turbulento comercializados no mercado brasileiro. A caracterização dos gotejadores seguiu os procedimentos das normas NBR ISO 9261. O experimento foi conduzido na Universidade Estadual Paulista (UNESP), Campus Botucatu, Departamento de Engenharia Rural. Para a realização do ensaio foram retirados ao acaso, de cada bobina, segmentos contendo 25 emissores. Na avaliação das características hidráulicas e técnicas dos gotejadores foram determinados os seguintes parâmetros: coeficiente de variação de fabricação; coeficientes da equação característica do gotejador; espessura da parede do tubo gotejador; diâmetro interno do tubo; espaçamento entre emissores; resistência à pressão com água na temperatura ambiente e a temperatura de $40{ }^{\circ} \mathrm{C}$; resistência do tubo a tração com tensão de $160 \mathrm{~N}$ e $180 \mathrm{~N}$; e ensaio de envelhecimento precoce. As análises dos tubos gotejadores mostraram um coeficiente de variação de fabricação inferior a 0,056 para todos os gotejadores $(0,07$ é limite máximo segundo a norma). Os expoentes $m$ da equação pressão versus vazão variaram de 0,431 a 0,575, classificando-os como não autocompensantes. Em relação a espessura da parede, o diâmetro interno dos tubos e o espaçamento entre os emissores foram constatados que todos os valores medidos estão dentro do exigido pela norma. Pelos resultados obtidos é possível afirmar que todos os produtos ensaiados ofertados no mercado brasileiro apresentaram excelente desempenho e qualidade.
\end{abstract}

Palavras-chave: Avaliação. Gotejador. NBR ISO 9261.

\section{HYDRAULIC AND TECHNICAL CHARACTERIZATION OF DRIPPERS NONSELF-COMPENSATING}

\begin{abstract}
This study aims to characterize and evaluate seven models of turbulent flow nonselfcompensating drippers available in the Brazilian market. The characterization of the emitters followed the procedures of the standards NBR ISO 9261. The experiment was conducted at UNESP University Estadual Paulista, Botucatu Campus, Department of Rural Engineering. For the execution of the test one randomly took from each coil, segments containing 25 emitters. In the evaluation of hydraulic and technical characteristics of emitters were determined the following parameters: coefficient of manufacturing variation, coefficients of the characteristic equation of the emitter, thickness of the tube wall, internal diameter, spacing between emitters, water pressure resistance at ambient temperature and $40{ }^{\circ} \mathrm{C}$, the tensile strength of the tube with a tension of $160 \mathrm{~N}$ and $180 \mathrm{~N}$, and premature aging testing. The analysis of the drip tapes showed a coefficient of manufacturing variation of less than 0.056 for all emitters. The exponents $(m)$ of the equation pressure versus flow rate ranged from 0.431 to 0.575 , classifying them as nonself-compensating. About the wall thickness, the internal diameter of the drip tapes and the spacing between emitters were found out that all measured values are within the required standard. From the results obtained, it can be stated that all products tested and available in the Brazilian market showed satisfactory performance and quality.
\end{abstract}

Keywords: Evaluation. Drip. NBR ISO 9261.

\footnotetext{
$\bar{*}$ Autor para correspondência

${ }^{1}$ Recebido para publicação em 31/03/2014; aceito em 10/08/2015.

${ }^{2}$ Professor, Departamento de Engenharia Rural, UNESP/FCAV, Jaboticabal (SP), CEP 14884-900; dalri@fcav.unesp.br

${ }^{3}$ Facultad de Agronomía y Zootecnia, Universidad Nacional de San Antonio Abad del Cusco, Perú.

${ }^{4}$ Professor, Departamento de Engenharia Rural, UNESP/FCAV, Jaboticabal (SP), CEP 14884-900.
} 


\section{INTRODUÇÃO}

A irrigação é uma técnica milenar que nos últimos anos vem se desenvolvendo acentuadamente, apresentando equipamentos e sistemas disponíveis para as mais distintas condições. $\mathrm{O}$ contínuo crescimento da população mundial vem exigindo uma agricultura competitiva que possibilite a produção de alimentos de melhor qualidade e em maior quantidade (MANTOVANI et al., 2012).

Em qualquer método de irrigação é fundamental que a distribuição de água às plantas seja realizada com a máxima uniformidade. $\mathrm{Na}$ irrigação localizada, essa distribuição se torna crítica porque as vazões fornecidas pelos emissores são pequenas e qualquer variação observada pode causar problemas, as vezes irreversíveis às culturas irrigadas, tanto pelo excesso de água aplicada quanto pelo déficit. O gotejamento tem se expandido consideravelmente nos cultivos de hortaliças, principalmente nas espécies sensíveis ao molhamento dos frutos (ANDRADE; BORGES JUNIOR, 2008).

Os emissores gotejadores constituem um dos principais componentes dos sistemas. Os materiais utilizados no processo de fabricação, bem como o próprio processo de fabricação, fazem com que os emissores de um mesmo modelo não sejam exatamente iguais entre si, e como consequência podem ocasionar diferentes vazões, mesmo quando trabalhando a mesma pressão de serviço (FRIZZONE; DOURADO NETO, 2003). Isso caracteriza os efeitos construtivos, os quais são avaliados pelo coeficiente de variação de fabricação (CVF).

A espessura da parede dos tubos usados como laterais varia muito, dependendo da durabilidade esperada e se o sistema será enterrado (ANDRADE e BORGES JUNIOR, 2008). Quanto mais delgadas forem as suas paredes menor o custo de produção. Os espaçamentos também são bem variados, de 0,1 $\mathrm{m}$ até $1,0 \mathrm{~m}$ entre emissores. Estes podem ser de fluxo turbulento e não apresentam dispositivos de compensação de pressão, ou autocompensantes, que dispõem de dispositivos de autorregulagem da vazão (PIZARRO, 1996; LOPEZ et al., 1997; GOMES, 1997).

Os gotejadores são emissores conectados às linhas laterais. É um dos componentes de maior importância nas instalações de irrigação localizada.
Toda dificuldade no seu projeto construtivo reside no seguinte problema: os emissores devem ser capazes de dissipar a pressão disponível e aplicar vazões pequenas, constantes e uniformes em pontos discretos sistematicamente localizados com o objetivo que as laterais e derivações sejam reduzidas (PIZARRO, 1996; BURT; STYLES, 2000; MOYA TALENS, 2002; BERNARDO et al., 2009). Por outro lado, a pressão de serviço dos emissores não deve ser alta, haja vista aumentar o consumo de energia do sistema, e também não muita baixa para não comprometer o desempenho hidráulico.

Para que os emissores tenham baixa vazão o diâmetro da seção de escoamento da água no emissor (labirinto) deve ser reduzido. Entretanto, há uma condição oposta a ser cumprida: o diâmetro da seção de escoamento do emissor deve ser o maior possível para reduzir o risco de entupimento, que se apresenta como um dos principais problemas no manejo dos sistemas de irrigação localizada. As respectivas condições tratadas são vistas pelos fabricantes de forma variada e como consequência existem no mercado muitos tipos de emissores.

Diante do exposto, objetivou-se neste trabalho caracterizar e avaliar técnica e hidraulicamente alguns tubos emissores não regulados, comercializados no mercado brasileiro e seguindo os procedimentos preconizados pela norma NBR ISO 9261 (ABNT, 2006).

\section{MATERIAL E MÉTODOS}

O presente trabalho foi conduzido na Faculdade de Ciências Agronômicas, na Universidade Estadual Paulista (UNESP), Campus Botucatu, Departamento de Engenharia Rural. A bancada de ensaios, composta por quatro linhas de teste (Figura 1), possui um depósito de água com capacidade de $500 \mathrm{~L}$ e uma motobomba para pressurizar as tubulações. Os tubos gotejadores com seus respectivos emissores possuem comprimento de $6 \mathrm{~m}$. Também fazem parte da bancada: válvulas de ar, manômetros, além de um filtro de tela metálica de 200 mesh.

Os manômetros foram aferidos antes do início dos testes de vazão dos tubos emissores. Fora utilizado manômetro analítico, classe A3, acoplado ao aparelho teste para realizar a respectiva avaliação. 


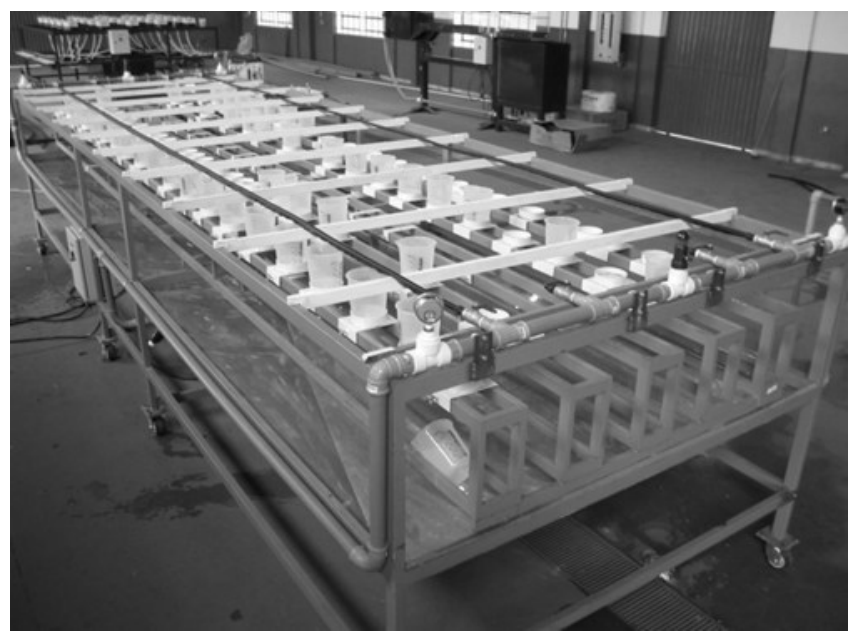

Figura 1. Vista geral da bancada de ensaios de emissores para gotejamento.

Foram utilizados os seguintes modelos de tubos emissores: Amanco Drip, Aqua Traxx, Tiran, Petro Drip, Golden Drip, Queen Gil e Chapin.

Na Tabela 1 são apresentados os tubos emis- sores selecionados e as principais características de cada um deles, obtidos tanto dos impressos encontrados nas bobinas como nos catálogos e páginas eletrônicas de cada empresa fabricante do tubo emissor.

Tabela 1. Características técnicas fornecidas pelos fabricantes dos tubos emissores avaliados na pesquisa.

\begin{tabular}{|c|c|c|c|c|c|c|c|c|c|c|c|}
\hline \multirow[t]{2}{*}{ Modelo } & \multirow{2}{*}{$\begin{array}{c}\mathrm{PN} \\
(\mathrm{kPa})\end{array}$} & \multicolumn{2}{|c|}{$\begin{array}{l}\text { Faixa de trabalho } \\
(\mathrm{kPa})\end{array}$} & \multicolumn{2}{|c|}{$\begin{array}{l}\text { Diâmetro do tubo } \\
(\mathrm{mm})\end{array}$} & \multirow{2}{*}{$\begin{array}{c}\mathrm{S} \\
(\mathrm{m})\end{array}$} & \multirow{2}{*}{$\begin{array}{c}\mathrm{E} \\
(\mathrm{mm})\end{array}$} & \multirow{2}{*}{$\begin{array}{l}\text { Vazão } \\
\left(\mathrm{L} \mathrm{h}^{-1}\right)\end{array}$} & \multicolumn{2}{|c|}{$\begin{array}{c}\text { Fórmula do } \\
\text { emissor }\end{array}$} & \multirow[t]{2}{*}{ CVF } \\
\hline & & mínimo & máximo & interno & externo & & & & $\mathrm{k}$ & $m$ & \\
\hline Petro Drip & 100 & 40 & 120 & 16,00 & ------ & 0,30 & 0,40 & 1,50 & 0,174 & 0,4611 & 0,04 \\
\hline Golden Drip & 100 & 30 & 120 & ------ & ------ & 0,30 & ----- & 1,50 & ------ & 0,53 & $<0,03$ \\
\hline Chapin & 70 & 30 & 100 & ------ & ------ & 0,30 & 0,375 & 1,24 & ------ & 0,54 & ------ \\
\hline Tiran & 100 & 50 & 300 & 14,10 & 16,10 & 0,50 & 1,00 & 2,00 & ------ & ------ & ------ \\
\hline Queen Gil & 70 & 30 & 100 & 16,50 & ----- & 0,30 & 0,20 & 1,20 & ------ & ------ & ------ \\
\hline Aqua Traxx & 70 & 30 & 100 & ------ & ------ & 0,305 & 0,20 & 1,27 & ------ & ------ & ------ \\
\hline Amanco Drip & 100 & 10 & 350 & ------ & 16,00 & 0,33 & 0,90 & 2,30 & ------ & ------ & ------ \\
\hline
\end{tabular}

PN: pressão nominal; S: espaçamento entre emissores; E: espessura da parede; e CVF: coeficiente de variação de fabricação.

Utilizou-se nos testes a água da Companhia de Saneamento Básico do Estado de São Paulo (SABESP), cuja análise química apresentou 2,1 mg $\mathrm{L}^{-1}$ de resíduos sólidos, estando dentro do limite aceitável de até $25 \mathrm{mg} \mathrm{L}^{-1}$, segundo a norma NBR ISO 9261. Durante o trabalho, a variação de temperatura, a qual era avaliada a cada 15 minutos, esteve entre $24{ }^{\circ} \mathrm{C}$ e $26{ }^{\circ} \mathrm{C}$, conforme prescreve a norma NBR ISO $9261\left(23^{\circ} \mathrm{C} \pm 3{ }^{\circ} \mathrm{C}\right)$.

Para cada bobina dos diferentes modelos de marcas comerciais foram retirados ao acaso 25 emissores para serem submetidos aos ensaios. Todas as avaliações do presente trabalho seguiram a mesma sequência preconizada pelas normas NBR ISO 9261 (ABNT, 2006).

Foram avaliadas as vazões de 25 emissores para cada tubo emissor, com quatro repetições, sendo utilizadas as pressões recomendadas pelo fabricante (Tabela 1). A vazão foi avaliada por pesagem e transformada em volume, sendo que o tempo para cada ensaio foi de 5 minutos.

Por meio dos dados observados da vazão do emissor (q) com a respectiva pressão (p) da água na tubulação foram ajustadas as equações características dos emissores gotejadores. Segundo a norma NBR ISO 9261, o modelo matemático que expressa a relação vazão versus pressão para emissores não regulados é do tipo potencial, ou seja, $\mathrm{q}=\mathrm{k} \mathrm{p}^{m}$, em que $\mathrm{k}$ é a constante e $m$ o expoente do emissor.

Com o auxílio de um micrômetro com precisão de $0,01 \mathrm{~mm}$ determinou-se a espessura da parede do tubo emissor em quatro pontos equidistantes em duas seções transversais ao comprimento do tubo, conforme Figura 2.

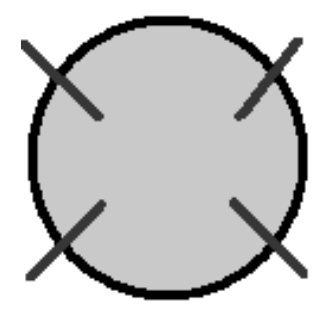

Figura 2. Secção transversal e posição dos pontos medidos nos tubos emissores. 
Para a determinação do diâmetro interno (Di) do tubo emissor fora inserido em uma das extremidades deste uma ferramenta cônica (Figura 3) com ângulo menor de $10^{\circ}$. Foram tomadas as devidas precauções com a finalidade de não danificar a abertura da mesma. Marcou-se na ferramenta o círculo feito por essa extremidade, medindo-se, posteriormente, com o auxílio de um paquímetro digital de $0,01 \mathrm{~mm}$ de precisão.

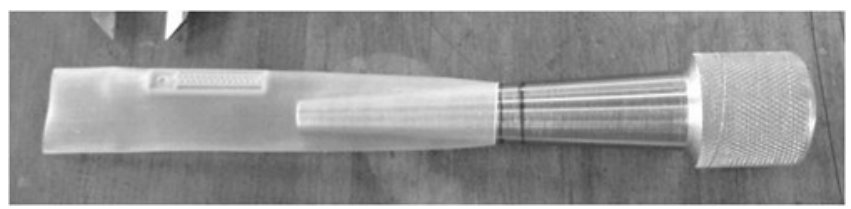

Figura 3. Ferramenta cônica utilizadas na determinação do diâmetro interno do tubo emissor.

O espaçamento entre emissores foi determinado com o auxílio de uma trena, com precisão de 1 $\mathrm{mm}$.

A resistência a pressão hidráulica em temperatura ambiente foi determinada obtendo da bobina de cada modelo um trecho do tubo emissor com cinco emissores. Esse segmento do tubo passou por duas avaliações. Uma à pressão de 1,2 vez a $p_{\max }$, e outra à pressão de 1,8 vez a $p_{\max }$, conforme se sugere a referida norma. Os tubos emissores ficaram gotejando por 60 minutos para cada pressão, frequentemente atentando para a retirada de todo o ar do sistema. A água foi mantida a uma temperatura entre 23 ${ }^{\circ} \mathrm{C}$ e $26{ }^{\circ} \mathrm{C}$, sendo reduzida lentamente a pressão de serviço para a pressão nominal, fazendo-se a medida da vazão por cinco minutos, com quatro repetições.

$\mathrm{Na}$ determinação da resistência à pressão hidráulica em condição de elevada temperatura $\left(40^{\circ} \mathrm{C}\right)$ delimitou-se um trecho com três emissores de cada modelo de tubo e, posteriormente, colocado em um recipiente e pressurizada com água a 1,2 da $p_{\max }$. A pressurização foi incrementada gradualmente tomando-se o cuidado para evitar o acúmulo de ar o interior do tubo emissor pressurizado. Logo após, o recipiente foi preenchido com água, a qual foi mantida aquecida a $40{ }^{\circ} \mathrm{C}$ por uma resistência blindada de $1600 \mathrm{~W}$, sendo esta temperatura monitorada com controle eletrônico. Detalhes podem ser observados na Figura 4. As mangueiras permaneceram em funcionamento e imersas por 60 minutos.

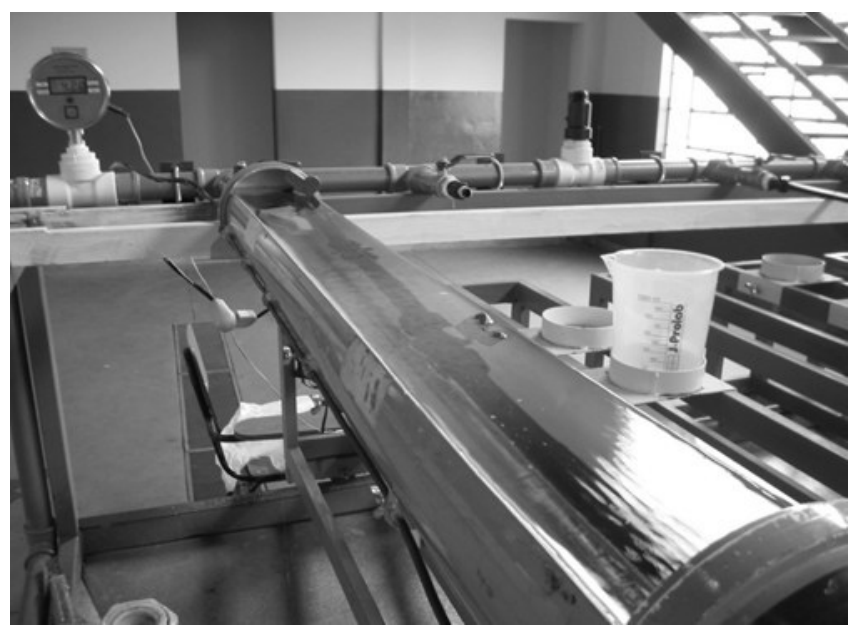

Figura 4. Recipiente plástico com resistência elétrica para aquecimento do tubo emissor.

Transcorridos 60 minutos, as mangueiras foram retiradas do recipiente e passaram por um repouso de 30 minutos em temperatura ambiente. E novamente feita a medição de vazão em pressão nominal, com quatro repetições e cinco minutos de coleta.

Também foi realizado o teste de resistência à tração a temperatura ambiente $\left(23^{\circ} \mathrm{C}\right)$, utilizando-se de dois trechos do tubo emissor de cada fabricante. Delimitaram-se dois pontos distantes entre si de 150 $\mathrm{mm}$. Em um dispositivo instalado no laboratório foram aplicadas duas tensões, $160 \mathrm{~N}$ e $180 \mathrm{~N}$. Tomouse o cuidado de incrementar a tração lentamente, de maneira a não danificar as amostras. A tração foi mantida por 15 minutos. Após esse tempo foram retiradas as mangueiras do dispositivo e avaliada a ocorrência de fissuras a fim de que o produto fosse considerado como reutilizável, no qual este deveria ter suportado a tensão de $180 \mathrm{~N}$ sem apresentar danos e, além disso, apresentar variação do alongamento do tubo menor que $\pm 5 \%$ entre as duas marcas analisadas.

Para o ensaio de envelhecimento precoce foram retiradas cinco amostras de $32 \mathrm{~cm}$ de cada tubo emissor avaliado. As amostras foram dobradas em forma de U, com dois planos perpendiculares diferentes, formando cantos vivos. Essa dobra ficou a pelo menos $5 \mathrm{~cm}$ da ponta do tubo emissor. Preparou -se uma solução concentrada com $200 \mathrm{~mL}$ do rea- 
gente ativo do tipo nonylphenoxy poly (ethyleneoxy) ethanol, (Renex) em $1800 \mathrm{~mL}$ de água (solução com $10 \%$ do reagente e $90 \%$ de água) em um béquer de vidro um pouco antes da realização do ensaio, agitando-se com agitador magnético por uma hora, com aquecimento a $70 \pm 2{ }^{\circ} \mathrm{C}$. Posteriormente, foram colocadas as cinco amostras dentro da solução (totalmente imersas) e depois de uma hora removidas. Analisou-se minuciosamente cada tubo emissor a fim de se detectar rachaduras visíveis na área das dobras. Foi utilizada para cada ensaio uma nova solução.

Os dados foram tabulados e as equações características do emissor gotejador tipo potencial obtidas com auxílio do software Excel 2007.

\section{RESULTADOS E DISCUSSÃO}

\section{Vazão (q) e Coeficiente de Variação de Fabrica- ção (CVF)}

$\mathrm{Na}$ Tabela 2 são observadas as diferenças percentuais entre as vazões fornecidas pelos fabricantes e as obtidas experimentalmente. Nota-se que nenhuma marca comercial superou os $7 \%$ de variação de vazão permitida pela norma, concluindo-se que os produtos avaliados estão dentro das especificações exigidas pela NBR ISO 9261. O modelo comercial Chapin, com pressão de $70 \mathrm{kPa}$, foi o que esteve mais próximo em relação a vazão informada pelo fabricante.

Tabela 2. Valores de vazão média dos emissores (q) medida e a variação em relação a vazão informada e valores informados e medidos do coeficiente de variação de fabricação (CVF).

\begin{tabular}{|c|c|c|c|c|c|c|}
\hline \multirow{2}{*}{ Modelo } & \multirow{2}{*}{$\begin{array}{c}\mathrm{PN} \\
(\mathrm{kPa})\end{array}$} & \multicolumn{2}{|c|}{ Vazão média q $\left(\mathrm{L} \mathrm{h}^{-1}\right)$} & \multirow{2}{*}{$\begin{array}{c}\text { Variação } \\
\text { observada }(\%) *\end{array}$} & \multicolumn{2}{|c|}{ CVF (\%) } \\
\hline & & Informada & Medida & & Informados & Medidos * \\
\hline Petro Drip & 100 & 1,50 & 1,47 & $-2,00$ & 4,4 & 3,8 \\
\hline Golden Drip & 100 & 1,50 & 1,43 & $-4,67$ & --- & 5,6 \\
\hline Chapin & 70 & 1,24 & 1,23 & $-0,81$ & 3,0 & 2,6 \\
\hline Queen Gil & 70 & 1,33 & 1,35 & $+1,50$ & --- & 2,8 \\
\hline Aqua Traxx & 70 & 1,14 & 1,12 & $-1,75$ & --- & 0,7 \\
\hline Tiran & 100 & 2,00 & 2,05 & $+2,50$ & 0,3 & 1,6 \\
\hline Amanco Drip & 100 & 2,30 & 2,26 & $-1,74$ & -- & 0,9 \\
\hline
\end{tabular}

PN: pressão nominal; * Variação permitida $\pm 7 \%$ (Norma NBR ISO 9261).

Em relação ao CVF, o efeito dos fatores construtivos dos tubos emissores, para todas as marcas avaliadas, esteve inferior a 7\% (limite máximo segundo a norma NBR ISO 9261). Portanto, pode-se inferir que todos os produtos encontrados no mercado brasileiro e avaliados neste trabalho podem ser considerados de ótima qualidade do ponto de vista do processo de fabricação. Os dados podem ser observados na Tabela 2 .

Um ótimo controle de qualidade no processo de fabricação dos emissores é uma das metas dos fabricantes, pois os emissores que apresentam valores elevados do CVF proporcionam alta variação da vazão entre eles (SOUZA et al., 2012), o que não é indicado para a irrigação localizada, principalmente quando se realiza a fertirrigação.

Prado et al. (2014), analisando as características hidráulicas do emissores, também encontraram valores do $\mathrm{CVF}$ dentro do limite máximo permitido pela norma de referência.

Os emissores da marca Aqua Traxx obtiveram o menor e melhor resultado de CVF, com um valor de $0,7 \%$. O valor do CVF medido do modelo Tiran foi superior ao informado pelo fabricante, sendo $0,3 \%$ o valor informado e $1,6 \%$ o valor medido.
Não foi possível fazer a comparação com os outros modelos, pois infelizmente os fabricantes não informaram os valores do CVF.

\section{Equação característica dos emissores - relação vazão versus pressão}

Em relação a variação da vazão dos emissores em função da pressão de entrada, esses tiveram suas vazões avaliadas em função de cinco diferentes pressões para a marca Chapin, seis pressões para as marcas Petro Drip, Golden Drip, Aqua Traxx, Queen Gil e Amanco Drip, e 7 pressões para a marca Tiran, cujos resultados estão apresentados nas Tabelas 3, 4 e 5, respectivamente.

Pode-se observar que as vazões dos emissores são muito sensíveis a variação na pressão, respondendo a vazão proporcionalmente à pressão aplicada. Segundo Lopez et al. (1997), os emissores considerados de fluxo turbulento possuem este comportamento. Como exemplo ilustrativo, o modelo Queen Gil apresentou variação da vazão de $42,52 \%$ quando a pressão variou em 71,43\% (70 a $120 \mathrm{kPa}$ ), e o modelo Petro Drip apresentou variação de vazão de $20,16 \%$ quando a pressão variou em 50\% (80 a $120 \mathrm{kPa})$. 
A. B. DALRI et al.

Tabela 3. Vazão média $\left(\mathrm{L} \mathrm{h}^{-1}\right)$ de 25 emissores das marcas Chapin, Queen Gil e Aqua Traxx em função da pressão.

\begin{tabular}{cccc}
\hline $\begin{array}{c}\text { Pressão } \\
(\mathrm{kPa})\end{array}$ & \multicolumn{3}{c}{ Vazão $\left(\mathrm{L} \mathrm{h}^{-1}\right)$} \\
\cline { 2 - 4 } & Chapin & Queen Gil & Aqua Traxx \\
\hline 30 & 0,862 & 0,869 & 0,709 \\
50 & 1,062 & 1,201 & ---- \\
55 & ---- & --- & 0,983 \\
70 & 1,230 & 1,350 & 1,124 \\
90 & 1,445 & 1,639 & 1,291 \\
100 & 1,584 & 1,742 & 1,360 \\
120 & ---- & 1,924 & 1,497 \\
\hline
\end{tabular}

Tabela 4. Vazão média $\left(\mathrm{L} \mathrm{h}^{-1}\right)$ de 25 emissores das marcas Petro Drip e Golden Drip em função da pressão.

\begin{tabular}{ccc}
\hline Pressão $(\mathrm{kPa})$ & \multicolumn{2}{c}{ Vazão $\left(\mathrm{L} \mathrm{h}^{-1}\right)$} \\
\cline { 2 - 3 } & Petro Drip & Golden Drip \\
\hline 40 & 0,986 & 0,909 \\
60 & 1,194 & 1,112 \\
80 & 1,334 & 1,259 \\
100 & 1,467 & 1,427 \\
120 & 1,603 & 1,545 \\
144 & 1,720 & 1,718 \\
\hline
\end{tabular}

Tabela 5. Vazão média $\left(\mathrm{L} \mathrm{h}^{-1}\right)$ de 25 emissores das marcas Tiran e Amanco Drip em função da pressão.

\begin{tabular}{ccc}
\hline Pressão $(\mathrm{kPa})$ & \multicolumn{2}{c}{ Vazão $\left(\mathrm{L} \mathrm{h}^{-1}\right)$} \\
\cline { 2 - 3 } & Tiran & Amanco Drip \\
\hline 50 & 1,501 & ---- \\
100 & 2,054 & 2,264 \\
150 & 2,484 & 2,751 \\
200 & 2,848 & 3,184 \\
250 & 3,205 & 3,541 \\
300 & 3,454 & 3,896 \\
350 & ---- & 4,194 \\
360 & 3,814 & ---- \\
400 & ---- & 4,463 \\
480 & ---- & 4,765 \\
\hline
\end{tabular}

A Tabela 6 apresenta as equações potenciais características dos respectivos tubos emissores. Observa-se que o expoente de fluxo $m$ que representa a sensibilidade do emissor à variação pressão varia de
0,431 para o Petro Drip a 0,575 para o Queen Gil. O expoente $m$, determinado para todos os modelos, apresentaram valores próximos de 0,5 , podendo ser classificados como emissores de fluxo turbulento.

Tabela 6. Equação do emissor dos tubos emissores estudados, sendo $\mathrm{q}\left(\mathrm{L} \mathrm{h}^{-1}\right)$ e $p(\mathrm{kPa})$.

\begin{tabular}{lc}
\hline Modelo & Equação do emissor \\
\hline Petro Drip & $\mathrm{q}=0,201 \mathrm{p}^{0,431}$ \\
Golden Drip & $\mathrm{q}=0,142 \mathrm{p}^{0,502}$ \\
Chapin & $\mathrm{q}=0,182 \mathrm{p}^{0,450}$ \\
Tiran & $\mathrm{q}=0,225 \mathrm{p}^{0,480}$ \\
Queen Gil & $\mathrm{q}=0,117 \mathrm{p}^{0,575}$ \\
Aqua Traxx & $\mathrm{q}=0,117 \mathrm{p}^{0,533}$ \\
Amanco Drip & $\mathrm{q}=0,260 \mathrm{p}^{0,470}$ \\
\hline
\end{tabular}

Pela NBR ISO 9261, quando o valor do expoente do emissor $m$ é maior do que 0,2, considera-se este emissor não autocompensante. Os valores de $m$ próximos a 0,5 indica que o regime de escoamento dos emissores é turbulento (PIZARRO, 1996; KELLER; BLIESNER, 1990; FRIZZONE et al., 2012), significando que as mudanças na vazão se relacionam com a raiz quadrada da pressão. Consequentemente uma variação na energia de pressão da ordem de $20 \%$ permite que a vazão dos emissores varie em torno de $\pm 10 \%$ em relação ao valor desejado. Andra- de (2005) encontrou $m$ igual a 0,494 e k igual a 0,154 para Golden Drip, valores muito próximos aos obtidos neste experimento. $\mathrm{O}$ valor do expoente da pressão da equação do emissor $(m)$ influencia diretamente no dimensionamento do comprimento das linhas laterais de irrigação (PRADO et al., 2014).

\section{Espessura da parede do tubo emissor}

A Tabela 7 apresenta os valores determinados da espessura de parede do tubo emissor. A norma 
NBR ISO 9261 recomenda que os fabricantes declarem esses valores arredondados em $0,05 \mathrm{~mm}$, e pelo critério desta norma, a espessura de parede do tubo emissor não pode ter nenhum dos quatro pontos com medida inferior a $90 \%$ da espessura declarada.

Tabela 7. Valores das espessuras de paredes dos tubos emissores, em mm.

\begin{tabular}{|c|c|c|c|c|c|c|c|c|c|}
\hline \multirow{2}{*}{ Marcas } & \multirow{2}{*}{$\begin{array}{c}\mathrm{E}^{*} \\
(\mathrm{~mm})\end{array}$} & \multicolumn{4}{|c|}{ Primeira seção } & \multicolumn{4}{|c|}{ Segunda seção } \\
\hline & & 1 & 2 & 3 & 4 & 1 & 2 & 3 & 4 \\
\hline Petro Drip & 0,40 & 0,44 & 0,45 & 0,44 & 0,41 & 0,47 & 0,45 & 0,44 & 0,42 \\
\hline Golden Drip & 0,40 & 0,42 & 0,43 & 0,45 & 0,46 & 0,45 & 0,47 & 0,44 & 0,43 \\
\hline Chapin & 0,40 & 0,48 & 0,46 & 0,51 & 0,49 & 0,47 & 0,48 & 0,46 & 0,49 \\
\hline Queen Gil & 0,20 & 0,23 & 0,28 & 0,26 & 0,24 & 0,25 & 0,28 & 0,23 & 0,24 \\
\hline Aqua Traxx & 0,20 & 0,20 & 0,21 & 0,23 & 0,21 & 0,22 & 0,23 & 0,22 & 0,20 \\
\hline Tiran & 0,90 & 1,02 & 0,99 & 0,98 & 1,01 & 1,04 & 1,02 & 1,03 & 1,01 \\
\hline Amanco Drip & 0,85 & 0,95 & 0,98 & 1,01 & 0,99 & 0,96 & 1,02 & 0,98 & 0,99 \\
\hline
\end{tabular}

* E: espessura fornecida pelo fabricante.

De modo geral, as espessuras determinadas em laboratório estão acima das fornecidas pelo catálogo dos fabricantes. Pode-se inferir que todas as marcas comerciais estão dentro das especificações da respectiva norma, para esse item.

\section{Diâmetro interno do tubo}

Em relação ao diâmetro interno dos tubos emissores a bobina ensaiada da marca Golden Drip, o diâmetro medido foi $16,31 \mathrm{~mm}$, com desvio de $0,31 \mathrm{~mm}$ sobre o diâmetro declarado no catálogo, de $16,00 \mathrm{~mm}$, sugerindo algum problema no controle de qualidade do fabricante. Entretanto, o erro foi para cima, favorecendo o consumidor ou o projetista para uma redução da perda de carga. $\mathrm{O}$ diâmetro interno medido não deve se desviar mais do que $\pm 0,3 \mathrm{~mm}$ do diâmetro fornecido pelo fabricante. Os valores medidos dos outros modelos estão dentro do aceitável pela norma e podem ser observados na Tabela 8 .

Tabela 8. Diâmetros internos fornecidos pelo fabricante e medidos dos tubos emissores avaliados.

\begin{tabular}{|c|c|c|c|}
\hline \multirow{2}{*}{ Produto } & \multicolumn{2}{|c|}{ Diâmetro interno (mm) } & \multirow{2}{*}{$\begin{array}{l}\text { Desvio } \\
(\mathrm{mm})^{*}\end{array}$} \\
\hline & Informado & Medido & \\
\hline Petro Drip & 16,00 & 15,78 & $-0,22$ \\
\hline Golden Drip & 16,00 & 16,31 & $+0,31$ \\
\hline Chapin & 16,00 & 16,04 & $+0,04$ \\
\hline Queen Gil & 16,50 & 16,34 & $-0,16$ \\
\hline Aqua Traxx & 16,00 & 16,24 & $+0,24$ \\
\hline Tiran & 14,10 & 14,38 & $+0,28$ \\
\hline Amanco Drip & 13,80 & 13,98 & $+0,18$ \\
\hline
\end{tabular}

*Aceitável \pm 0,3 (Norma NBR ISO 9261).

\section{Espaçamento entre emissores}

Em relação ao espaçamento entre os emissores, pode-se observar nas medições realizadas e apresentadas na Tabela 9 que todos os tubos emissores analisados estão dentro da faixa de tolerância. Se- gundo a norma brasileira NBR ISO 9261, os espaçamentos entre os emissores não devem desviar mais do que $5 \%$ do espaçamento declarado pelo fabricante. Os resultados desta pesquisa confirmam a boa qualidade dos produtos comercializados no Brasil.

Tabela 9. Espaçamento entre emissores dos tubos analisados.

\begin{tabular}{|c|c|c|c|c|c|c|}
\hline \multirow{2}{*}{ Marcas } & \multirow{2}{*}{$\begin{array}{c}\text { Espaçamento } \\
\text { Informado (mm) }\end{array}$} & \multicolumn{3}{|c|}{ Valores medidos } & \multirow{2}{*}{$\begin{array}{l}\text { Valor médio } \\
\text { medido }(\mathrm{mm})\end{array}$} & \multirow{2}{*}{$\begin{array}{l}\text { Desvio* } \\
(\%)\end{array}$} \\
\hline & & 1 & 2 & 3 & & \\
\hline Petro Drip & 300 & 303 & 305 & 304 & 304,00 & $+1,67$ \\
\hline Golden Drip & 300 & 300 & 295 & 298 & 297,67 & $-1,67$ \\
\hline Chapin & 300 & 309 & 309 & 309 & 309,00 & $+3,00$ \\
\hline Queen Gil & 300 & 295 & 296 & 295 & 295,33 & $-1,67$ \\
\hline Aqua Traxx & 305 & 301 & 302 & 301 & 301,33 & $-1,31$ \\
\hline Tiran & 500 & 505 & 501 & 500 & 502,00 & $+1,00$ \\
\hline Amanco Drip & 330 & 344 & 332 & 343 & 339,67 & $+4,24$ \\
\hline
\end{tabular}

*Maior desvio com relação ao valor mais alto. 


\section{Resistência à pressão}

Durante o teste de pressurização não foram constatados sinais de danos aos tubos emissores ensaiados. Os tubos emissores de qualquer fabricante não romperam e nenhuma aparência visual de rompimento (formação de bolhas) ocorreu durante essa etapa. Após reduzir lentamente a pressão para o valor nominal e aguardar por 3 minutos foi realizado o teste de vazão, no qual todos os tubos emissores de todas as marcas comerciais não apresentaram variações significantes de vazões.

Para os produtos reutilizáveis, o critério de pressão se torna mais exigente, $1,8 \mathrm{vez}$ a pressão máxima. Nesse critério, todos os tubos emissores foram considerados reutilizáveis, até aqueles com menor espessura de parede. Consequentemente, pode -se inferir que todos os produtos podem ser classificados como reutilizáveis para essa pressão máxima, de acordo com a norma NBR ISO 9261.

\section{Resistência à pressão hidráulica submetida a ele- vada temperatura $\left(40^{\circ} \mathrm{C}\right)$}

Após a pressurização dos tubos emissores o recipiente com o sistema de aquecimento foi ligado e preenchido com água. Observou-se nos ensaios que a temperatura da água do recipiente variou de $30^{\circ} \mathrm{C}$ a $42{ }^{\circ} \mathrm{C}$. Todos os modelos de emissores apresentaram comportamento excelente quanto a essa característica, como observado na Tabela 10, concluindo-se que a temperatura não afeta a vazão desses modelos de emissores analisados. $\mathrm{O}$ modelo que apresentou a maior variação foi o Petro Drip, com valor igual a $0,314 \%$, ou seja, após o aquecimento houve pequena redução da vazão, porém muito inferior ao preconizado pela norma NBR ISO 9261, que é de $10 \%$. Os modelos de Tiran e Amanco Drip foram os menos susceptíveis a variação de temperatura.

Tabela 10. Valores de vazão antes e depois do ensaio em água a $40^{\circ} \mathrm{C}$ e as variações encontradas para as marcas Petro Drip, Golden Drip, Chapin, Queen Gil, Aqua Traxx, Tiran e Amanco Drip.

\begin{tabular}{lccc}
\hline \multirow{2}{*}{ Modelo } & \multicolumn{2}{c}{ Vazão $\left(\mathrm{L} \mathrm{h}^{-1}\right)$} & \multirow{2}{*}{$\begin{array}{c}\text { Diferença } \\
(\%)\end{array}$} \\
\cline { 2 - 3 } & antes & após & $-0,314$ \\
Petro Drip & 1,382 & 1,377 & $-0,214$ \\
Golden Drip & 1,399 & 1,396 & 0,227 \\
Chapin & 1,468 & 1,471 & $-0,098$ \\
Tiran & 2,050 & 2,048 & 0,229 \\
Queen Gil & 1,748 & 1,752 & 0,209 \\
Aqua Traxx & 1,115 & 1,117 & 0,060 \\
Amanco Drip & 2,214 & 2,215 & 0,060 \\
\hline
\end{tabular}

\section{Resistência do tubo à tração}

Nos ensaios de resistência à tração, o modelo Queen Gil, mesmo tendo espessura relativamente baixa $(0,20 \mathrm{~mm})$, foi o que apresentou menor variação, com $0,67 \%$, sendo que o modelo Aqua Traxx com a mesma espessura não passou no ensaio, haja vista que a distância entre os pontos variou acima do limite permitido nas duas tensões testadas. Esse modelo apresentou $18,67 \%$ de variação entre os pontos medidos para a força de $160 \mathrm{~N}$ e $32,00 \%$ para a força de 180 N. Os demais modelos responderam à exigência da norma, conforme observado na Tabela 11 .

Tabela 11. Distância entre os pontos submetidos a forças de tração aplicadas aos tubos emissores.

\begin{tabular}{|c|c|c|c|c|c|c|}
\hline \multirow{3}{*}{ Marca } & \multicolumn{3}{|c|}{ Força aplicada de $160 \mathrm{~N}$} & \multicolumn{3}{|c|}{ Força aplicada de $180 \mathrm{~N}$} \\
\hline & \multicolumn{3}{|c|}{ Distância entre dois pontos (mm) } & \multicolumn{3}{|c|}{ Distância entre dois pontos (mm) } \\
\hline & Antes & Depois & Variação \% & Antes & Depois & Variação \% \\
\hline Petro Drip & 150 & 153 & 2,00 & 150 & 154,0 & 2,67 \\
\hline Golden Drip & 150 & 153 & 2,00 & 150 & 153,5 & 2,33 \\
\hline Chapin & 150 & 152 & 1,33 & 150 & 152,0 & 1,33 \\
\hline Queen Gil & 150 & 151 & 0,67 & 150 & 151,0 & 0,67 \\
\hline Aqua Traxx & 150 & 178 & 18,67 & 150 & 198,0 & 32,00 \\
\hline Tiran & 150 & 151 & 0,67 & 150 & 152,5 & 1,67 \\
\hline Amanco Drip & 150 & 151 & 0,67 & 150 & 151,0 & 0,67 \\
\hline
\end{tabular}

\section{Ensaio de envelhecimento precoce}

Nos testes de resistência dos tubos emissores ao envelhecimento precoce ou fadiga ambiental, pode-se observar no resultado do ensaio (número de dobras com falhas) que em todos os tubos emissores testados nenhum apresentou rachadura visível.

\section{CONCLUSÕES}

Os coeficientes de variação de fabricação determinados nos tubos emissores avaliados ficaram abaixo de 0,07 , o que é recomendado pela norma referencial do presente estudo.

$\mathrm{O}$ expoente $m$ da equação característica vari- 
ou de 0,431 e 0,575 , sendo todos os emissores classificados como de fluxo turbulento.

Os produtos ensaiados, de acordo com a norma NBR ISO 9261, e ofertados no mercado brasileiro apresentaram excelente desempenho e qualidade.

O material usado como matéria-prima na fabricação dos tubos emissores é de boa qualidade.

\section{REFERÊNCIAS}

ANDRADE, L. Avaliação hidráulica e de resistência a tração de dois modelos de mangueiras gotejadoras. 2005. 76 f. Dissertação (Mestrado em Agronomia: Áre de Concetração em Irrigação e Drenagem) - Universidade Estadual Paulista, Botucatu, 2005.

ANDRADE, C. de L. T de; BORGES JUNIOR, J. C. F. Seleção do método de irrigação. In: ALBUQUERQUE, P. E. P. de; DURÃES, F. O. M. (Ed.). Uso e manejo de irrigação. Brasília: EMBRAPA, 2008. cap. 7, p. 317-399.

ABNT. ASSOCIAÇÃO BRASILEIRA DE NORMAS TÉCNICAS. NBR ISO 9261: Equipamentos de irrigação agrícola. Emissores e tubos emissores. Especificação e métodos de ensaio. São Paulo, 2006.

BERNARDO, S.; SOARES, A. A.; MANTOVANI, E. C. Manual de Irrigação. 8 ed. Viçosa, MG: UFV, 2009. $625 \mathrm{p}$.

BURT, C. M.; STYLES, S. W. Drip and microirrigation for trees, vines, and row crops. San Luis Obispo: The Irrigation Training \& Research Center, 2000, $261 \mathrm{p}$.

FRIZZONE, J. A.; DOURADO NETO, D. Avaliação de sistemas de irrigação e análise econômica. In: MIRANDA, J. H. de; PIRES, R. C. de M. (ed.). Irrigação. Jaboticabal: FUNEP, 2003. v. 2, cap. 15, p. 573-652.

FRIZZONE, J. A. et al. Microirrigação: gotejamento e microaspersão. Maringá: Eduem, 2012. 356 p.

GOMES, H. P. Engenharia de irrigação: Hidráulica dos sistemas pressurizados, aspersão e gotejamento. 2 ed. Ver. Ampl. Campina Grande, 1997. 390 p.

KELLER, J.; BLIESNER, R. D. Sprinkle and trickle irrigation. New York: van Nostrand Reinhold, 1990. $652 \mathrm{p}$.

LOPEZ, R. J. et al. Riego localizado. 2 ed. Madrid: Ediciones Mundi Prensa, 1997. 405 p.

MANTOVANI, E. C.; BERNARDO, S.; PALARETTI, L. F. Irrigação: princípios e métodos. 3 ed. Viçosa, MG: UFV, Imprensa Universitária, 2012.
$355 \mathrm{p}$.

MOYA TALENS, J. A. Riego Localizado y Fertirrigación. 3 ed. Madrid: Ediciones Mundi Prensa, 2002. 534 p.

PIZARRO, F. Riegos Localizados de alta Frecuencia (RLAF): goteo, microaspersión, exudación. 3 ed. rev.ampl. Madrid: Mundi Prensa, 1996. 513p.

PRADO, G. do; NUNES, L. H.; TINOS, A. C. Avaliação técnica de dois tipos de emissores empregados na irrigação localizada. Revista Brasileira de Agricultura Irrigada, Fortaleza, v. 8, n. 1, p. 12-25, 2014.

SOUZA, W. J. et al. Irrigação localizada subsuperficial: gotejador convencional e novo protótipo. Revista Brasileira de Engenharia Agrícola e Ambiental, Campina Grande, v. 16, n. 8, p. 811-819, 2012. 